\title{
ARTIGO DEFINIDO ITALIANO ORIGEM E EVOLUÇÃO
}

\section{Luigi Castagnola}

A língua latina, que deu origem à língua italiana, não fazia uso do artigo definido.

Eis um verso latino de Fedro:

"Ad rivum eundem lupus et agnus venerant", cuja tradução, em prosa italiana, pode ser a seguinte:

"Il lupo e l'agnello erano venuti ad un medesimo rivo". Como se vê, a língua italiana introduziu o artigo definido:

Latim: "Iupus" - "agnus"

Italiano: "il lupo" - "I" agnello".

A filologia nāo tem dificuldade para demonstrar que o artigo definido italiano teve origem das diversas formas do adjetivo demonstrativo latino "ille", por enfraquecimento das mesmas.

Adjetivo demonstrativo latino:

Sing. masc.: il (le)

(i) lum

Plural masc.: (ii) li

$$
\text { i (IIi) }
$$

Sing. fem.:

(i) $\mathrm{la}$

Plural fem.:

(il) lae
Artigo definido italiano:

il

lo

li

i

la

le.

Já no período clássico da lingua latina, a linguagem popular costumava colocar o adjetivo demonstrativo "ille" diante do substantivo: "ille homo; illa domus; illi servi". Destarte o substantivo ficava determinado de modo melhor.

As pesquisas lingüísticas de Rohlfs, relativas aos dialetos italianos da atualidade, evidenciam que, ainda hoje, em determinados lugares da Tosr'na, vigoram as expressões seguintes: "ill'acqua", ill'uva, ill'aia, ill'orto, ill'anni, ill'erbe, ill'osse; ill'ago, ill'amío, ill'ove" (1).

(1) Cf. G. ROHLF5, Grammafies storiea della lingua italiana a doi suol dialotti, vol. II, Morfologia, Einaudi, Torino, 1967, p. 103. 
O encurtamento do adjetivo demonstrativo, que deu origem às formas do artigo definido italiano, processou-se lentamente ao longo dos séculos que viram a desagregaçāo da língua clássica latina e a morosa organização da língua italiana. Este período de tempo, que viu o surgir demorado, também, das outras línguas neolatinas, pode ser compreendido entre os séculos $\mathrm{V}$ e XII.

Quanto à língua italiana, o uso do artigo definido já se encontra em documentos do século XI. Eis uns documentos:

1 - Ista cartula est de caput coctu/ille adiuvet de illu rebottu qui mal consiliu li mise in corpu (1087).

II - Me acuso de lu corpus Domini, K'io indignamente lu accepi... Pregonde la sua sancta misericordia e la intercessione de li suoi sancti (Séc. XI).

O século XII apresenta abundância maior de documentos, em que o uso do artigo definido é mais desenvolvido. Eis alguns dêstes documentos:

1 - E mea fo l'opra Nicolao Scolptore (1135).

11 - Salv' a lo vescovo senato,/lo mellior c'unque sia nato...

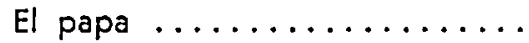

$\mathrm{L}^{\prime}$ apostolico romano ...........

Da che 'I mondo fue pagano....

cui bendicente bascio la mano (Séc. XII).

III - Et set ce fosse impedementu varcante, lu 'impedementu sia complitu... (1186).

IV - Dua Casteldart havi li nostri bona part; / i lo zetà futto intro lo flumo d'Ard,/e sex cavaler de Tarvis li plui fer/ con se duse li nostri cavaler (1193).

Ficou destarte evidenciado, através da citação de documentos, que a lingua italiana, nascida de longo e confuso processo evolutivo do latim popular, conhecia e fazia uso do artigo definido já nos séculos XI e XII. E claro que as formas dêste artigo estavam ainda longe daquela pureza lingüística que têm hoje; também seu uso era muito oscilante. O labor de progressivo polimento realizou-se durante os séculos da história da língua italiana, desde o século XIII até, pràticamente, o século XIX. Quanto à uniformidade do emprêgo do artigo masculino, é de se dizer que foi ela alcançada, com respeito a certos casos, sòmente ao alvorecer do século $X X$, como veremos mais adiante.

Com relação às formas e ao uso do artigo definido feminino 
as coisas foram relativamente fáceis, desde o comêço. Conforme os bons autores do passado e do presente, e conforme as recomendaçōes dos gramáticos de maior autoridade, as formas e o uso do artigo definido feminino podem-se compendiar do modo seguinte:

$\begin{array}{ll}\text { Singular } & \text { Pl } \\ \text { la } & \text { le } \\ l^{\prime} & l^{\prime}\end{array}$

No singular, usa-se a forma apostrofada / $/$ ' diante de vogal, e a forma não apostrofada /la/ diante de consoante.

"La casa, la madre, la zia, la rosa, la scuola".

"L’anima, l'erba, l'unghia, l'orsa, l'italiana".

Entretanto é de se notar que, no passado, bons autores costumaram, também, não apostrofar o artigo /la/ diante de vogal. Dante Alighieri, na "Vita Nuova", que pode ser considerado como o primeiro livro escrito em prosa artística da língua italiana, emprega estas expressões:

"La ebrietà, la imaginazione, la infallibile veritate".

Machiavelli, mais do que qualquer outro, costuma não apostrofar: "La edificazione, la occasione, la espugnazione, la ltalia, la infelicità, la ambizione, la origine, la ignavia, la insolenzia, la obedienzia".

Della Casa, no "Galateo", usa "la invidia, nella usanza, alla avarizia".

Fòscolo, nas "Ultime lettere di Jàcopo Òrtis", usa as duas formas: "la Isabellina; I'Isabellina".

No plural, conforme as recomendaçōes dos bons gramáticos, a forma $/ \mathrm{l} /$ pode ser empregada sòmente diante de palavra que começa por $/ \mathrm{e} /$. No entanto se encontra a forma apostrofada também diante de palavras que começam por outra vogal, tanto em autores antigos como em escritores modernos. Esste emprêgo, no entanto, é raro.

Machiavelli: "l'arme Orsine".

Dante: "I'ore, l'altre persone, l'altre donne".

Petrarca: "I' altre donne, l'ore, l'ire".

Fòscolo: "l'acque, l'aure, l'urne, l'itale glorie".

D'Annunzio: "dolci l'acque, l'ultime opere". 
No uso das diversas formas do artigo definido masculino, a uniformidade foi alcançada em épocas bastante tardias, como acima se salientou. Ainda no comêço dêste século havia escritores de grande fama que usavam, vez por outra, o artigo /il/, no singular, e /i/, no plural, diante de palavra que começa por $/ z /$.

Carducci: "il Zanella, il Zanardini".

Panzini: "i zamponi”.

Panzini fêz ainda uso do artigo /i/ diante do digrama /gn/.

O seguinte paradigma abrange tôdas as formas do artigo definido masculino, atualmente usadas pelos modernos escritores da Itália e ensinadas pelos melhores gramáticos:

$\begin{array}{ll}\text { Singular } & \text { Plural } \\ / \mathrm{il} / \mathrm{/} / \mathrm{l} / \mathrm{li} / \\ / \mathrm{lo} / \mathrm{l} / & / \mathrm{gli} /, / \mathrm{gl}^{\prime} /\end{array}$

1) A forma / /o/ usa-se diante de:

a) palavra que começa por $|z|$ : "lo zio, lo zero, lo zucchero";

b) palavra que começa por /s/ impura, isto é, seguida por ouira consoante: "lo scudo, lo spillo, lo stuolo, lo stretto, lo sguardo";

c) palavra que começa pelo digrama /gn/: "lo gnocco, lo gnostico";

d) palavra que começa pelo digrama /ps/: "lo psicologo, lo psichiatra";

e) palavra que começa pelo digrama $/ \mathrm{pn} /$ : "lo pneumatico, lo pneuma";

f) palavra que começa por $/ x /$ : "lo xilografo, lo xenofobo";

g) palavra que começa por vogal que tem valor de consoante: "lo iato, lo iugoslavo".

É perciso, no entanto, salientar que sòmente diante das palavras indicadas em a), b), c) é constante o emprêgo de $/ 10 /$. Diante das outras categorias de palavras acima indicadas emprega-se também a forma / il/, sendo que êste último costume tende a se generalizar. O que torna bem mais simples o uso do artigo definido masculino.

As palavras que começam por $/ x /$ são raras, em italiano, e tendem a trocar $/ x /$ por /5/: "il silografo". 
2) A forma $/ 1 \%$ usa-se diante de palavra que começa por vogal: "I'anno, l'altare, l'otre, l'ultimo, l'italiano, l'empio".

3) A forma /il/ é usada diante de palavra que começa por consoante que não seja /z/: "il banco, il palazzo, il libro, il cibo, il collo".

4) A forma aferética /'l/ é usada pouco pelos modernos autores. Usa-se quando a palavra que precede termina por vogal: "da ', a \%, ne 'I".

D'Annunzio: "de 'I ciel, co 'I gelido, a 'I bacio, da I verde".

Carducci: "pe 'I tacito, su "I prato, tra "I Celio".

Os antigos autores faziam muito uso desta forma aferética, que hoje é sentida como forma arcaica ou poética.

No plural, usa-se:

1) /Gli/ quando a palavra seguinte começa por: $/ z /, / s /$ impu$\mathrm{ra}, / \mathrm{gn} /, / \mathrm{ps} /,|\mathrm{pn} /| x \mid$, , e por vogal com valor de consoante. E também diante de palavras que começam por vogal, contanto que não seja $/ i /$.

"Gli zii, gli zeri, gli scudi, gli spilli, gli gnomi, gli gnocchi, gli psicologi, gli pneumatici, gli xilografi, gli iugoslavi, gli alberi, gli otri, gli umili, gli economi".

Também aqui é preciso salientar que o emprêgo de /gli/ é constante diante de $/ \mathrm{z} /, / \mathrm{s} /$ impura, $/ \mathrm{gn} /$ ). Nos demais casos pode-se empregar também /i/. O uso parece mostrar a tendência a preferir esta segunda forma. Diante de vogal, que não seja /i/, usa-se sempre a forma $/ \mathrm{gli} /$.

2) Diante de palavras que começam por /i/, usa-se a forma apostrofada: /gl'/: "gl'italiani, gl'inglesi, gl'ingegneri, gl'infermieri". É preciso notar, porém, que os autores não observam sempre mordicus esta norma de apostrofar o artigo /gli/ diante de palavras que começam por /i/. Gioberti: "gli Italiani"; D'Azeglio: "gli Italiani".

3) Emprega-se /i/ diante de palavra que começa por simples consoante:

"i bambini, i baci, i salti, i denti, i voti, i tori; $i$ soldati, i soldi".

Anigamente havia outra forma para o plural masculino: /li/. Encontraremos esta forma na rápida exemplificação histórica que faremos a seguir. Atualmente esta forma é usada, raras vêzes, por algum escritor; mas tem sabor arcaico e o uso generalizado a baniu 
das boas letras. Carducci ainda fêz uso dela: "Forse Francesca temprò qui li ardenti

\section{Occhi al sorriso?"}

Nāo é de se crer, depois de ter exposta a doutrina do atual uso do artigo definido italiano, que as formas do presente tenham sido usadas também no passado. Pelo contrário. Até foram usadas formas que desapareceram por completo, e pertencem, portanto, ùnicamente à cultura filológica. Por exemplo, as formas do plural masculino: /e/, /ei/, /e'/ e a forma do singular masculino: /el/.

Citaremos exemplos de autores de todos os séculos e através destas citações ficará evidenciada a trajetória oscilante pela qual passou o artigo definido italiano, antes de chegar à fase uniformizada dos tempos atuais.

Século XIII (II Duecento).

São Francisco de Assis: "lo frate sole"

Giácomo da Lentíni: "lo vostro amore, lo bel viso".

Guittone D'Arezzo: "lo valore e 'I poder".

Guido Guinizelli: "lo sol, lo cor, lo foco, lo ciel, gli occhi, i miei desiri".

"Il Novellino": "el favolatore, il giullare, il villano, lo villano, lo figlivolo, l'uscio, lo 'mperadore, i suoi nemici, li figlivoli, el padre, li anni, el soldano".

lacopone da Tódi: "lo tuo figliuolo, lo figlio, li ladroni, el figlio, li pié, lo dolor, "l cor, i miei compagni, l'odio". lo spirto".

Guido Cavalcanti: "li fiori, li occhi, li verdi, gli occhi, gli augelli,

Neste século predomina, no singular, a forma $/ \mathrm{lo} / ;$ é muito usada, também, a forma aferética $/ 1 /$. No plural predomina a for$\mathrm{ma} / \mathrm{li} /$.

\section{Século XIV (II Trecento).}

Dante Alighieri: "lo nono giorno, lo cielo, lo mio nascimento, lo spirto, il spirto, l'altro, il nome, lo die, lo loco, il mar, il sol, il cor, li quali, li spiriti, li termini, li nomi, li occhi, gli occhi, gli spiriti".

Petrarca: "lo qual, che 'I fren, quando 'I pianeta, l'ultimo volo, lo stil, il peccar nostro, il fine, il dí, il mondo, il sacco, i suoi, gli stecchi, i nervi, i rai, e' laghi, li alti penseri, li occhi, li sdegni, li ebrei, gli atti". 
Boccaccio: "el riso, el desio, el vago sguardo, lo mio disio, il tempo, il zio, il caldo, l'altrui, gli orrevoli, l'uscio, li padri, li quali, i duo monti, i cavalli, gli Iddii, gl'Iddii".

Sacchetti: "gli ambasciadori, li quali, lo abate, l'altro, e 'l detto, i discepoli, il vino, gli oficiali, il signore, li maestri, il zappator, i gozzi".

Com relação ao século precedente, 0 artigo /lo/ perdeu terreno. Usa-se muito, especialmente por Petraarca, a forma aferética $/$ ' $I /$; há muita liberdade de escolha entre /il/ e /el/. No plural a liberdade no uso das diversas formas é ainda maior, mas as mais usadas são /i/ e /li/. Diante de / $z$ / é usado o artigo /il/.

\section{Século XV (II Quattrocento).}

Poliziano: "el cor, el paradiso, lo sforzo, el riso, el vago crino, lo glorioso tuo fratel, il primo fiore, el fascio, il giovinetto, gli artigli, i monti".

Lorenzo il Magnifico: "il cor, lo incauto pastor, el cor, el ballo, el grande amor, el rio, li ammirabili, gli alti monti, i vecchi".

M.M. Boiardo: "el pregio, el gran desio, il ciel, lo imperatore".

Palmieri: "el zelo, l'avaro, il fatto, i viventi, gli altri, i figliuoli".

Pulci: "il Danese, e 'I gentil, l' abate, i mesi, gli anni, gli altri, i galli, l'oste, lo scandaglio, gli abissi".

Masuccio Salernitano: "el quale, il quale, lo spirito, el ditto corpo, el maestro morto, el frate, il frate".

Sannazaro: "lo ombroso faggio, lo eccelso pino, il restante, l'altare, li più chiari, i fiumi, i faggi, li quali, li tesi inganni, i capi".

A forma /el/ é muito usada; podemos dizer que predomina. As formas /li/ e /lo/ são pouco usadas. No plural predomina a forma $/ \mathrm{i} /$.

Como é sabido, no século XV os Humanistas hostilizaram o vulgar e pretendiam voltar ao uso do latim. Essa pretensão anacrônica foi abandonada pelos literatos de Quinhentos.

Século XVI (II Cinquecento).

Este século foi muito fecundo no campo das letras, e a questāo da língua alvoroçou todos os arraiais literários. Pietro Bembo, no terceiro livro das Prose della volgar lingua (1525), fêz uma exposição das partes principais da gramática da língua vulgar, e com sua auto- 
ridade lingüística influiu sôbre os escritores posteriores. Ludovico Ariosto, na última edição de seu famoso poema "L'Orlando Furioso" (1532), obedece quase sempre às normas traçadas por Bembo relativas ao uso do artigo definido, abandonando as formas /el/ e /e/. que mudou para /il/ e / i/. Houve quem afirmasse serem as formas /el/ e /e/ não só um vício, mas um horrendo e espantoso monstro da língua italiana.

Feitas estas considerações, vejamos, através de exemplos, como os Quinhentistas usaram o artigo definido.

Machiavelli: "el Geta, el sole, el populo, il lione, il duca, lo essere, lo andare, lo assalto, e' Romani, il re, gli stati, gli vomini, li vomini, l'altro, li portamenti, gli ambiziosi, li populi, li Spartani, li Romani, e' potenti, i quali, i meschini, i disegni, i Pazzi".

Guicciardini: "il Parlamento, l'appello, to affermare, il re, lo effetto, lo sforzo, lo Imperio, il popolo, i miseri, i Fiorentini, i provvedimenti, gli Spagnuoli, gli altri".

Ariosto: "il cardellino, l' alto valor, lo scritto, lo scudo, il fracasso, il brando, gli Etiopi, li appetiti, li strani liti, i denti, i panni, gli amori, l'ispido ventre, gli occhi".

Della Casa: "i zotici, gli spiacevoli, lo stridere, lo strepito, lo spirito, l'vomo, gl'idioti, gli occhi, lo spagnuolo, lo italiano, i bugiardi, i domestici, li Napolitani".

Castiglione: "il confe, il specchio, lo amante, l'ordine, gli animi, il sforzare, i gesti, i meriti, li più eccellenti".

Tasso: "il dolce gelo, il ver, il velo, l'animo, l'ufficio, l'oro, lo scudo, e 'I zelo, lo stuolo, il zelo, gl'imperi, gl'insulani, gli anni, gli sciti, gli estremi, i topi, i fantasmi, g!i augelli".

De Machiavelli a Tasso o caminho percorrido é deveras importante. Machiavelli é um irregular no emprêgo dos artigos; talvez nenhum escritor tenha usado, com tanta liberdade, tão numerosas e variadas formas de artigo definido. $E$ já no emprêgo das formas do artigo feminino, notamos como êle trilhou sendas que hoje chamaríamos de libertárias. Feita exceção de Machiavelli, vemos que há uma inclinação sensibilíssima para o abandono de /el/ e /lo/, no singular; o próprio Machiavelli, nas obras da velhice, vai-se conformando ao uso geral. Não foi pouca a influência exercida pelos gra. máticos sôbre os escritores e, em Tasso, encontramos já o artigo definido usado como o usam os bons literatos italianos da atualidade. $O$ que tira à sua língua aquela asperidade que encontramos nos auto- 
res que o precederam e the confere um rítmo doce e macio. Ariosto e Tasso, sem dúvida possivel, exerceram influência notável, quanto ao emprêgo e às formas do artigo definido, sôbre as gerações dos séculos posteriores. No entanto, também em Quinhentos, diante de $/ z /$ vigora o uso de /il/ e não de /lo/.

Século XVIII (II Seicento).

Marino: "il mento, il lido, il suono, l'aspro, l'infelice, lo scoppio, lo spirto, gli errori, gli ardori, i monti, i bellicosi".

Chiabrera: "l'arido, l'orecchio, il suicidio, lo stil, lo sguardo, i barbari, i vanti, gli aperti, gli scirocchi".

Boccalini: "il poema, il Tasso, il calcio, l'agnello, l'avvocato, gli occhiali, gl'ingegni, gl'intelletti, gli scritti, gli steccati, gli strapazzi, gli Italiani, i mali, i giudici, i préncipi, gli errori".

Testi: "il viaggio, il vino, il cielo, lo stomaco, l'vomo, l'ozio, gli Spagnuoli, gli affetti, i frutti, i denti, gli infelici, i zaffiri".

G. Galilei: "il medesimo, il signore, il poeta, l'opposto, l'istesso, l'incendio, i piombi, i corpi, i pezzi, gli effetti, gli altri, gli vomini, gli ingegni".

Continua o uso de /il-i/ diante de / $z$. Há casos de /li/, mas raros. De qualquer forma, os autores, seguindo os vestígios de Ariosto e Tasso, aproximam-se do uso atual, no emprêgo do artigo definido.

\section{Século XVIII (II Settecento).}

Neste século o uso do artigo tende cada vez mais a uniformizarse, aproximando-se das formas atuais. Encontra-se ainda /li/ no plural, em lugar de $/ \mathrm{i} /$, mas é raro. Também raro é o emprêgo de $/$ lo/ em lugar de /il/. Vico escreve: "Lo che; per lo mezzo; lo ingegno". Ainda Muratori escreve: "il zelo", e outros também. A forma aferética /'l/ é bastante usada. Parini escreve: "lo dio dell'armi", "lo ingegno". Alguma liberdade no uso do artigo definido, no entanto, deve ser considerada licença poética.

Século XIX (L’Ottocento).

Foi êste o século que viu a unificação da ltália, a gloriosa época do Ressurgimento Italiano. A imprensa e os escritores de tôda a península tiveram grande parte no movimento político que unificou as diversas regiōes italianas. Mas êsse fato político, tão importante para a Itália, fêz com que homens de todos os paises italianos se misturassem, fêz com que fôsse usada uma mesma língua na administração 
pública. Coisa não fácil, se se considerar que, em cada região, se falavam dialetos bastante diferentes. Tudo isto acarretou, como é claro, consequiências também no campo lingüístico. Daí decorreu uma certa confusão no uso puro da língua italiana. Este fato despertou novamente a questão da língua, na qual teve uma parte de primeira ordem Alexandre Manzoni. Com seu romance "I Promessi Sposi", Manzoni não só deu à língua italiana a sua obra-prima em prosa, mas também o modêlo de uma prosa pura e popular ao mesmo tempo. De sorte que ninguém exerceu maior influência sôbre a prosa italiana do que o grande lombardo, a partir do ano da publicação de "I Promessi Sposi". Este famoso livro ofereceu a todos os Italianos o modêlo de uma língua doce, fluente, elegante e popular ao mesmo tempo. No emprêgo do artigo definido houve ainda certa oscilação, especialmente na primeira metade do século. Fòscolo escreve ainda: "il scintillante, i stemmi, i zeffiri". A forma do plural /li/, especialmente na prosa administrativa, diante de vogal ("li articoli, li vomi$\mathrm{ni}^{\prime \prime}$ ), diante de /s/ impura ("li strilli, li stinchi"), aparece ainda de vez em quando. Leopardi, no "Epistolario", usa "il zio, i zii"; mas nas últimas cartas (de $1834 \mathrm{em}$ diante) passa a escrever habitualmente "lo zio, gli zii". De sorte que, no fim do século XIX, o emprêgo do artigo definido é, pràticamente, fixo e uniformizado. Os casos esporádicos que ainda se encontram, contrários ao uso comum, não têm mais influência sôbre o público.

Século XX (II Novecento).

Na atualidade, o uso do artigo definido é fixo e constante. Os modernos meios de comunicação (rádio, jornal, revista, televisão, livro) por certo facilitam a generalização dêsse uso, que é outrossim ensinado nas escolas e inculcado pelos bons gramáticos.

Algum autor de renome, por exemplo, D'Annunzio, usa ainda a forma aferética / $/ /$. Mas pode ser considerada uma maneira estilística pessoal, com que o super-homem D’Annunzio pretendia distinguir-se dos simples mortais. 